\title{
Review of: "“They talk to me like a person" Experiences of people with opioid use disorder in an injectable opioid agonist treatment (iOAT) program: A qualitative interview study using interpretive description"
}

\author{
Dimy Fluyau ${ }^{1}$ \\ 1 Emory University
}

Potential competing interests: The author(s) declared that no potential competing interests exist.

The author conducted an interview, a survey, to explore client experiences in an injectable opioid agonist treatment program. The paper may benefit some work to present findings at a more academic level. The author may define the type of study, define the method more appropriately. The write-up of the findings does not fit a scholarly article. A table may help simplify and conceptualize sentences to a framework that can be presented in a table.

Methods

In the methods section, the author wrote that the research team conducted a secondary thematic analysis of qualitative interviews with iOAT clients. What was the primary research about? What type of study was the primary one? What type of data was the primary one?

In the research method, the term interpretive description needs further explanation.

Data Analysis

In data analysis, what is Inductive coding? What type of software was used? What type of statistical method was used to interpret data?

Findings

The presentation of findings needs to be structured with results of similar research. The use of sentences in the findings section may be in a table. The main finding may be reported as a conceptual framework in the findings section. One can conceptualize the sentence below, for example, in a table. "I have a place where I can come where I feel safe. Where I feel like people care. Where people don't look at me like a junkie. Like an addict. They look at me like a person. They talk to me like a person. I like that we have that family structure. It's like that family-orientated kind of set up. I like that... It helped me get my life back, and... I like that no matter what's going on, they'll always make time for you. Really. It's - doesn't matter what's going on, if you're having a bad day, they're there for you. No matter what." [P07]".

\section{Discussion}

The author delineates the main findings and discusses the limitations of the study.

Conclusion

It is informative. 
